\title{
Light and drugs: the photochemistry of fluoroquinolone antibiotics
}

\author{
Elisa Fasani, Angelo Albini, Mariella Mella, Michela Rampi, \\ and Federico Barberis Negra \\ Dipartimento di Chimica Organica, Università di Pavia, \\ v. Taramelli 10, 27100, Pavia, Italy
}

\begin{abstract}
Light-related adverse side-effects of drugs are now an important source of concern. In order that the mechanism underlying to such effects is recognised, an in-depth photochemical study must be carried out. The case of some fluoroquinolone antibiotics (norfloxacin, enoxacin, lomefloxacin) is discussed as a representative example. These drugs undergo heterolytic fragmentation of the $\mathrm{C}-\mathrm{F}$ bond leading to aryl cations. Quantum yields in neutral water range from 0.001 to 0.5 depending on the charge transfer degree of the $\pi \pi^{*}$ state. Lower values are obtained at both acidic and basic $\mathrm{pH}$. There is indication that the excited state involved in most of this reactions is a relatively long-lived triplet. The aryl cations undergo intra- or intermolecular reactions, and are presumably involved in the reported photo-toxic effect.
\end{abstract}

\section{INTRODUCTION}

Many drugs, just as non-pharmaceutically active compounds, are significantly photoreactive. Indeed, this is no recent observation. Pasteur noticed the photolability of quinine in 1846 and the photochemistry of drugs was already recognised to be sufficiently important to deserve industry-sponsored studies in the twenties. However, until recently the matter has received only limited attention, also because it is generally feasible to protect the drug preparation from light through an appropriate package. As a result, the available knowledge is quite sparse. All Pharmecopoeias mention that some drugs have to be protected from light, but this cautionary note is accompanied only by qualitative and incomplete information. The number of reports devoted to this subject in specialised journals is growing, but remains low. However, the situation has changed recently due to three causes.

First. There have been several cases of promising drugs which have been discarded at a late stage during the development process due to a too high photolability. The development of a new drug is very expensive and this calls for more attention to the photochemical properties of a molecule early in the development.

Second. More sensitive analytical methods are now available and the standard of purity required has become more and more stringent. This has led to formulation by ICH (International Committee of Harmonisation) of internationally accepted Guidelines for Drugs Photostability, which have been implemented since January 1998.

Third. Significant phototoxic effects have been ascertained for several drugs, as well as for cosmetic products and sunscreens. The effect (skin erythema, oedema, ocular opacity, etc.) is usually monitored through standard methods. This allows to estimate the risks of the use of a drug in combination with exposure to light. However, in order to really understand why a drug is phototoxic, the clinical test must be complemented by a thorough photochemical and photobiological study. The molecular processes which a drug undergoes after absorption of UV or visible light must be ascertained and the structure not only of the endphotoproducts but also of the reactive intermediates such as radicals or ions must be demonstrated.

Although the dimension of the problem is not yet recognised by the general public, the percentage of drugs in common use having a significant phototoxic effect is far from negligible. The increased awareness of this problem led in the last years to the multiplication of specialised meetings (Oslo 1995, Pavia 1997, Washington 1999) and of books [1,2].

\section{THE PHOTOCHEMISTRY OF DRUGS}

The photochemistry of drugs does not differ, in general terms, from that of the other molecules, and is therefore rationalised (and can be roughly predicted) according to the same generalisations that are useful in organic photochemisty. In particular, it is generally easy to indicate in each molecule some feature that is likely to make it liable to photodecomposition. This does not lead to an exact prediction of the photochemical behaviour of a drug, since it is difficult to estimate how the various physical and chemical processes compete. At any rate, the type and efficiency of a photochemical reaction depends not only on the structure, but also on the experimental or applicative conditions. The last point is particularly important for drugs and may lead to a significant different photochemistry in vitro and in vivo. At any rate, several chemical functions are expected to introduce photoreactivity in a molecule, and their presence must alert about possible photoactivity.

Typical such functions are (see Scheme 1):

(a) The $\mathrm{C}=\mathrm{C}$ double bond. Such molecules usually undergo $E / Z$ isomerisation and may undergo polar addition or cycloaddition quite easily. 

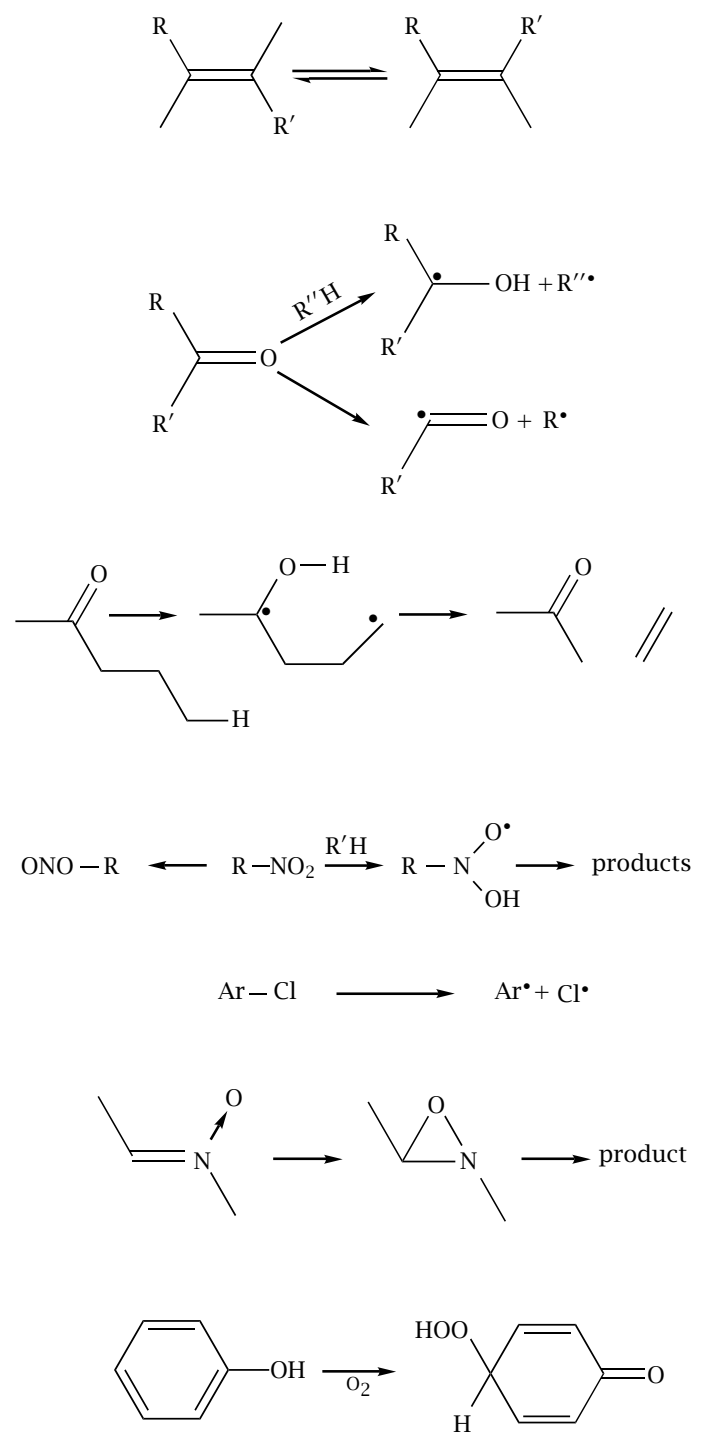

Scheme 1

(b) The $\mathrm{C}=\mathrm{O}$ double bond. The carbonyl function behaves as an electrophilic radical in the excited state and is easily involved in inter- or intramolecular hydrogen transfer processes or fragmentation reaction.

(c) Among aromatics, nitro derivatives resemble ketones in their radical reactivity and chlorides often undergo homolytic or heterolytic dechlorination.

(d) Among heterocycles, five-membered rings usually undergo easy rearrangements and $\mathrm{N}$-oxides are involved in electrocyclic reactions.

(e) Products containing weak $\mathrm{C}-\mathrm{H}$ bonds, such as is the $\alpha-\mathrm{C}-\mathrm{H}$ in amines, alcohols, ethers, sulfides may be involved in oxidative or degradative processes, often related to the activation of oxygen by photosensitisation.

It is immediately apparent from the inspection of the above list, that a very large fraction, if not the majority of commonly used drugs contain some of these functions and thus are expected to be light-sensitive. This does not necessarily indicate that all of such drugs are photolabile under applicative conditions, since, e.g., not all of them absorb significantly ambient (natural or indoor) light $(\lambda>c a .300 \mathrm{~nm})$ or because absorption of light in concentrated solutions, in suspensions or in solids might be unefficient.

Most of the available data on the photochemistry and photophysics of molecules have been obtained in dilute solutions in organic solvents, since these are the most convenient conditions for mechanistic studies. However, the behaviour in aqueous solution or suspension or in the solid state, viz under conditions nearer to those under which drugs are used, may be quite different. To take a textbook example, benzophenone triplet, apparently the most extensively investigated excited state, is a short-lived species in an organic solvent (e.g., $\tau$ ca. $0.3 \mu \mathrm{s}$ in ethanol) where it reacts via hydrogen abstraction, while its lifetime increases by two order of magnitude in water, where this molecule is almost photostable. More or less extended compilations of the known photoreactions of drugs are available in the literature [1-4].

\section{THE PHOTOCHEMISTRY OF FLUOROQUINOLONES}

We refer here some recent results about the photochemistry of fluoroquinolones, since these seem in many ways representative of the problems encountered in this field. This important class of antibiotics has evolved from non fluorinated quinolones initially used as drugs, such as oxolinic acid and nalidixic acid. Following elaboration has shown that quinolones bearing 7-amino and 6-fluoro substituents are particularly convenient from the therapeutic point of view and compounds of this group (third generation quinolones) presently represent the almost totality of prescribed quinolones.

Typical examples are: norfloxacin, enoxacin, ofloxacin, lomefloxacin, cyprofloxacin (Scheme 2). The introduction of a fluorine atom is a common practice in drug design and is often associate to an improvement in the efficacy while avoiding undesirable side effects because of the great chemical inertness of the carbonfluorine bond which imparts a wellcome stability to the molecule. As it will appear from the following, however, this does not hold in photochemical conditions, where in fact it is the carbon-fluorine bond which is labilised. The impetus to this study comes from a number of reports appeared in recent years documenting the phototoxic potential of such drugs, which will be commented on in the next section. In previous works, it was known that fluoroquinolones are weak oxygen sensitisers and further that some of these derivatives underwent photo-initiated degradation of the alkylamino side-chain in 7. This is the case for ofloxacin [5] as well as for cyprofloxacin [6]; for the latter molecule, the occurring also of a photo-dimerisation has been proposed. In 1997, however, both Chignell [7] and De Guidi and Monti [8] reported formation of fluoride ions by irradiation of enoxacin, lomefloxacin and sparfloxacin in phosphate buffer. 
<smiles>CCn1cc(C(=O)O)c(=O)c2cc3c(cc21)OCO3</smiles>

oxolinic acid<smiles>CCn1cc(C(=O)O)c(=O)c2ccc(C)nc21</smiles>

nalidixic acid<smiles>CCn1cc(C(=O)O)c(=O)c2cc(F)c(N3CCNCC3)nc21</smiles>

orfloxacin

enoxacin<smiles>CC1COc2c(N3CCN(C)CC3)c(F)cc3c(=O)c(C(=O)O)cn1c23</smiles><smiles>CCn1cc(C(=O)O)c(=O)c2cc(F)c(N3CCNC(C)C3)c(F)c21</smiles>

lomefloxacin

Scheme 2

Our aim was to isolate the photoproducts, in order to have a firm evidence about their structure, and to carryout the irradiation under well defined conditions in water. Fluoroquinolones are aminoacids and thus are amphoteric substances. At neutral $\mathrm{pH}$ the dominant form is a zwitterion, below $\mathrm{pH} 5-5.5$ is a cation, over $\mathrm{pH} 9$ is a anion (see Scheme 3 ). Thus the photochemistry is expected to be $\mathrm{pH}$ dependent. Our preparative study<smiles></smiles><smiles>CCn1cc(C(=O)O)c(=O)c2cc(F)c(N3CC[NH2+]CC3)cc21</smiles>

Scheme 3 gave final evidence that the main process is defluorination (accounting for $>95 \%$ of the decomposed material) and allowed the isolation of the thus formed product(s) [9].

Norfloxacin may be considered the simplest example of third generation quinolones. Irradiation of norfloxacin at $\mathrm{pH} 7.2$ led to a slow decomposition $(\Phi=$ $0.06)$. The only process was defluorination $\left(\Phi_{-\mathrm{F}}=\Phi_{\mathrm{dec}}\right)$ and involved solvolysis at position 6 (Scheme 4) [10].

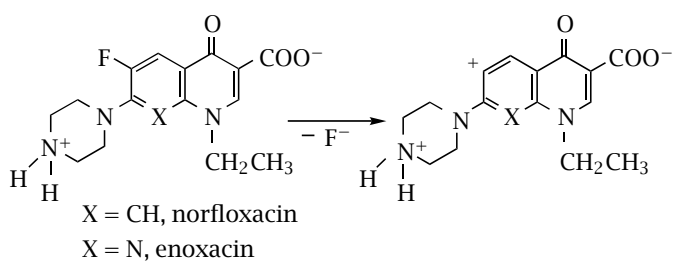

Scheme 4

Exactly the same type of process, with a somewhat increased efficiency, was observed with the 1,8 naphthyridine analogue enoxacin. Such photoinduced solvolysis took place also at $\mathrm{pH} 4.5$ and 10, although with lower quantum yield. The only reaction occurring at pH 1 was side chain decomposition. With both drugs, the reaction was quenched to a considerable extent by oxygen, and reasonably involved the triplet state. Defluorination is no common process in the photochemistry of aromatics. Two mechanisms can be considered for this process: a $\mathrm{SN}_{2}$ addition-elimination mechanism as demonstrated for some alkoxy nitro benzenes and related derivatives; or a $\mathrm{SN}_{1}$ mechanism as recently proposed for some halogenated aryl ethers and anilines [11,12].

For various reasons the latter mechanism seems attractive. Obviously a homolytic fragmentation of the $\mathrm{C}-\mathrm{F}$ bond is out of question on thermodynamic grounds (because the energy of this bond, $477 \mathrm{KJ} \mathrm{mole}^{-1}$, is larger than that of the excited singlet, ENX $333 \mathrm{KJ} \mathrm{mole}^{-1}$, and a fortiori of the triplet state, $278 \mathrm{KJ} \mathrm{mole}^{-1}$ ), while heterolytic fragmentation appears viable when one takes into account the internal charge transfer character of the excited state of push-pull aromatics such as these quinolones are. This hypothesis is supported by the observed structurereactivity relationship. An electron-attracting moiety increases the defluorination quantum yield while an electron-donating group strongly decreases it apparently by balancing the partial positive charge of the fluorine bearing carbon. Also while changing the $\mathrm{pH}$ in the interval 4.5-10 affects the efficiency but not the course of the reaction, a further decrease to $\mathrm{pH} 1$ suppresses defluorination. In the 4.5-10 range the equilibria involve the $4^{\prime}$ nitrogen, whereas at $\mathrm{pH} 1$ protonation is directly on the $\pi$ system (see Scheme 3 ) and precludes the charge transfer required for defluorination. Under this condition the only photochemistry observed is inefficient side-chain decomposition, as mentioned above.

The photochemistry of lomefloxacin is more 
<smiles>[H][Y18]1([H])CCN(c2c(F)cc3c(=O)c(C(=O)[O-])cn(CC)c3c2F)CC1C</smiles>

Scheme 5

complicated, and we were intrigued by the complex chemistry occurring. This is a 6,8-difluoro derivative and the reaction occurring was found to be again defluorination, in this case selectively from position 8 . The reaction is less dependent on $\mathrm{pH}$ and practically unaffected by the presence of oxygen. The same reaction was observed in the whole pH-range 1-10 and the product was always the derivative resulting from insertion into the N-ethyl chain (see Scheme 5). The quantum yield for decomposition was higher than in the previous cases. In part this is due to the presence of a further electron-withdrawing group and in part to a different reason connected with the selectivity.

We think that the selectivity is due to the better stabilisation of the cation in position 8. For this cation, a mesomeric formula conserving the aromaticity of the pyridone moiety can be considered, while this is not the case for the cation in position 6 (see Scheme 6). The<smiles>[H][Y]1([H])CCN(c2cc3c(cc2F)c(=O)c(C(=O)[O-])cn3CC)C[C@@H]1C</smiles><smiles>[H][Y17]1([H])CCN(c2ccc3c(=O)c(C(=O)[O-])cn(CC)c3c2F)CC1</smiles>

Scheme 6

different behaviour of lomefloxacin on one hand and of norfloxacin and enoxacin on the other is reminescent of the dual chemistry observed by Schuster [13] for aryl cations generated by nitrogen elimination from diazonium salts in trifluoroethanol. The relation between electronic structure and reactivity has not been completely characterised for these elusive species. A number of studies in matrix have been devoted to establish which is the ground state. There are two possibilities: a singlet state and a triplet state. It has been concluded that the ground state of aryl cations is a triplet when it is substituted with an electron-donating group in para position. When the substituent is an electronwithdrawing group the ground state is a singlet. However this does not necessarily determine which will be the chemical reaction in the fluid. A singlet state cation with a vacant $\sigma$ orbital is expected to add nucleophiles, while a triplet state shares an electron from the $\pi$ cloud and resembles an aryl radical. Thus it is expected to enter in homolytic processes (see Scheme 7).

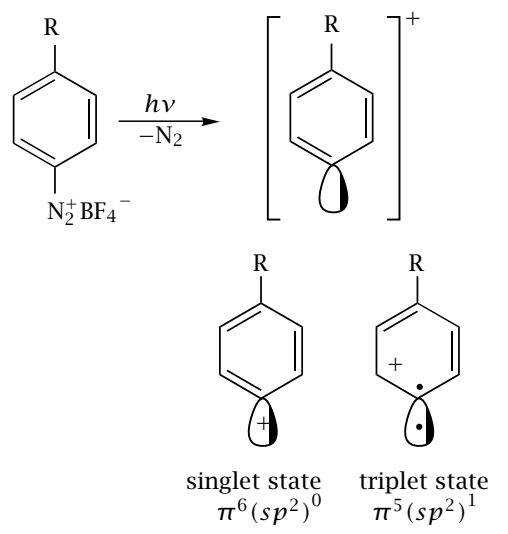

Scheme 7

In Schuster's experiments in trifluoroethanol, the 4dialkylamino aryl cation gave the corresponding ether, viz the product expected from the singlet, despite the fact that this cation has a triplet ground state. On the other hand the 4-benzoyl cation abstracted hydrogen from the solvent, reasonably via the triplet, despite the fact that it has a singlet ground state.

Thus, the observed intramolecular insertion in lomefloxacin can be envisaged as a two steps radical reaction (hydrogen abstraction-biradical coupling). This peculiar behaviour may be rationalised on the bases of the different mesomeric equilibrium. For the cation in position 8 a $\pi$ mesomer is more important than for a cation in position 6. (In this structure the charge is on the nitrogen and position 8 has a carbene character, possibly as a triplet.)

The main conclusions of our photochemical work are as follows:

(1) Fluoroquinolones undergo heterolytic defluorination (quantum yield varies from 0.001 to 0.5 depending on the internal charge transfer degree of the $\pi \pi^{*}$ excited state).

(2) The reaction is typical of the zwitterion and is much less efficient under both basic and acidic conditions.

(3) There is indication that excited state involved in most of these reactions is a relatively long-lived triplet. 


\section{RELATION BETWEEN PHOTOCHEMISTRY AND PHOTO-TOXIC EFFECT}

Dermal photosensitisation by fluoroquinolones has been documented in mice and in humans by several clinical studies and includes various forms of dermathosis [14-16], scarification of the skin [17], onycolysis [18] and subcorneal eruption [19]. Moreover, photomutagenic and phototumorigenic effects have been also reported in 1997 [20,24].

At the beginning of our study, the current hypothesis for rationalising the phototoxic effect invoked some form of activated oxygen as the key species. However, this hypothesis does not fit with the evidence that has been recently provided.

(1) Fluoroquinolones do activate oxygen producing both singlet oxygen and superoxide anion, but these are inefficient processes and at any rate ranging the fluoroquinolones according to their efficiency as oxygen sensitisers gives a list not correlating with their order of phototoxicity [22].

(2) On the other hand, the phototoxicity is parallel to photoreactivity.

(3) In particular, it has been known for some time [22] that the presence of a halogen $(\mathrm{F}$ or $\mathrm{Cl}$ ) at position 8 strongly enhanced the phototoxicity of quinolone drugs and, as seen above, a 8-halogeno atom is highly photolabile. As an example, recently Chignell showed that 6,8-difluoro derivatives such as lomefloxacin and fleroxacin are 10-fold more efficient in generating single strand DNA breaks after UVA irradiation than the less photoreactive 6-fluoro derivatives such as norfloxacin [23, 24].

The fact that the photoinduced defluorination occurs in the same order as photoreactivity while this is not the case for the ability of these drugs to activate oxygen, coupled with the formation of highly reactive intermediates (aryl cations) in the defluorination process, suggests that the adverse biological effect may be related to irreversible reactions involving covalent binding of the aryl cation to some cell component.

\section{REFERENCES}

[1] H. H. Tønnesen (ed.), Photostability of Drugs and Drug Formulations, Taylor \& Francis, London,
1996.

[2] A. Albini and E. Fasani (eds.), Drugs: Photochemistry and Photostability, The Royal Society of Chemistry, Cambridge, 1998.

[3] J. V. Grenhill and M. A. McLelland, Prog. Med. Chem. 27, 51.

[4] G. M. J. Beijersbergen van Henegouwen, Adv. Drug. Res. 29 (1997), 79.

[5] Y. Yoshida, E. Sato, and R. Moroi, Arzneim. Forsch. 43 (1993), 601.

[6] E. M. Tiefenbacher, E. Haen, B. Przybilla, and H. Kurz, J. Pharm. Sci. 83 (1994), 463.

[7] L. Martinez, G. Li, and C. F. Chignell, Photochem Photobiol. 65 (1997), 599.

[8] S. Sortino, G. De Guidi, S. Giuffrida, S. Monti, and A. Velardita, 67 (1998), 167.

[9] E. Fasani, M. Mella, D. Caccia, S. Tassi, M. Fagnoni, and A. Albini, Chem. Commun. 1329 (1997).

[10] E. Fasani, A. Profumo, and A. Albini, Photochem. Photobiol. 68 (1998), 666.

[11] G. Zhang, P. Wan, and A. Albini, Chem. Commun. (1998), 19.

[12] A. P. Durand, R. G. Brown, D. Worrall, and F. Wilkinson, J. Chem. Soc., Perkin Trans. 2 (1998), 365.

[13] S. M. Gasper, C. Devadoss, and G. B. Schuster, J. Am. Chem.Soc. 117 (1995), 5206.

[14] J. Ferguson, J. Photochem. Photobiol. 62 (1995), 954.

[15] P. Ball and G. Tillotson, Drug Saf. 13 (1995), 343.

[16] P. S. Lietman, Drugs 45 (1995), (sup. 3).

[17] Y. Kurimaji and M. Shono, Contact Dermatitis 26 (1992), 5.

[18] R. Baran and P. Brun, Dermatologica 173 (1986), 185.

[19] E. D. Shelley and W. B. Shelley, Cutis 42 (1988), 24.

[20] G. Kecak, F. Urbach, and H. Urwyler, J. Photochem. Photobiol. B 37 (1997), 169.

[21] H. J. Reavy, N. J. Traynor, and N. K. Gibbs, Photochem. Photobiol 66 (1997), 368.

[22] J. M. Domagala, J. Antimicrob. Chemother. 33 (1994), 685.

[23] L. Martinez, R. H. Sik, and C. F. Chignell, Photochem. Photobiol. B 67 (1998), 399.

[24] L. Martinez and C. F. Chignell, Photochem. Photobiol. B 45 (1998), 51. 


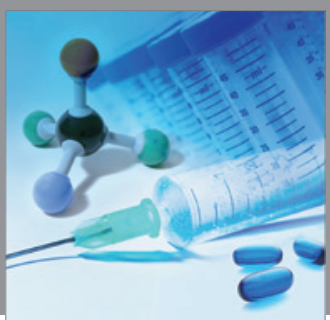

International Journal of

Medicinal Chemistry

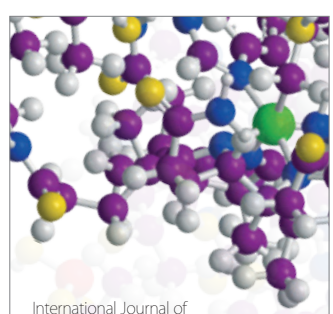

Carbohydrate Chemistry

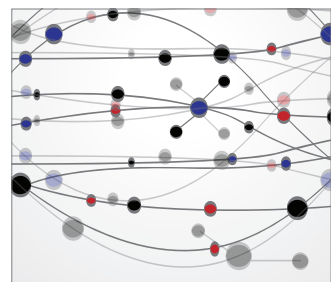

The Scientific World Journal
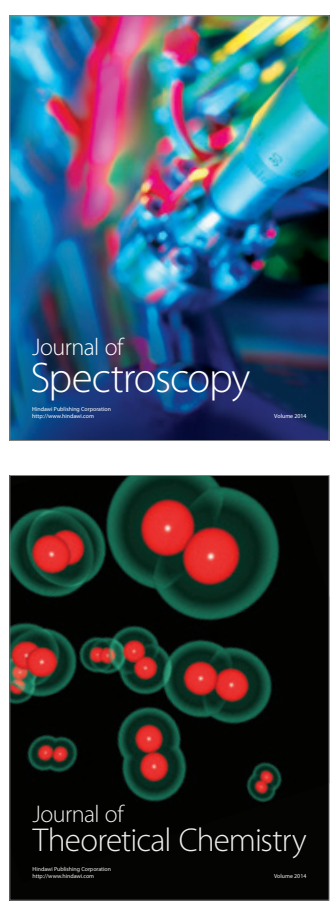
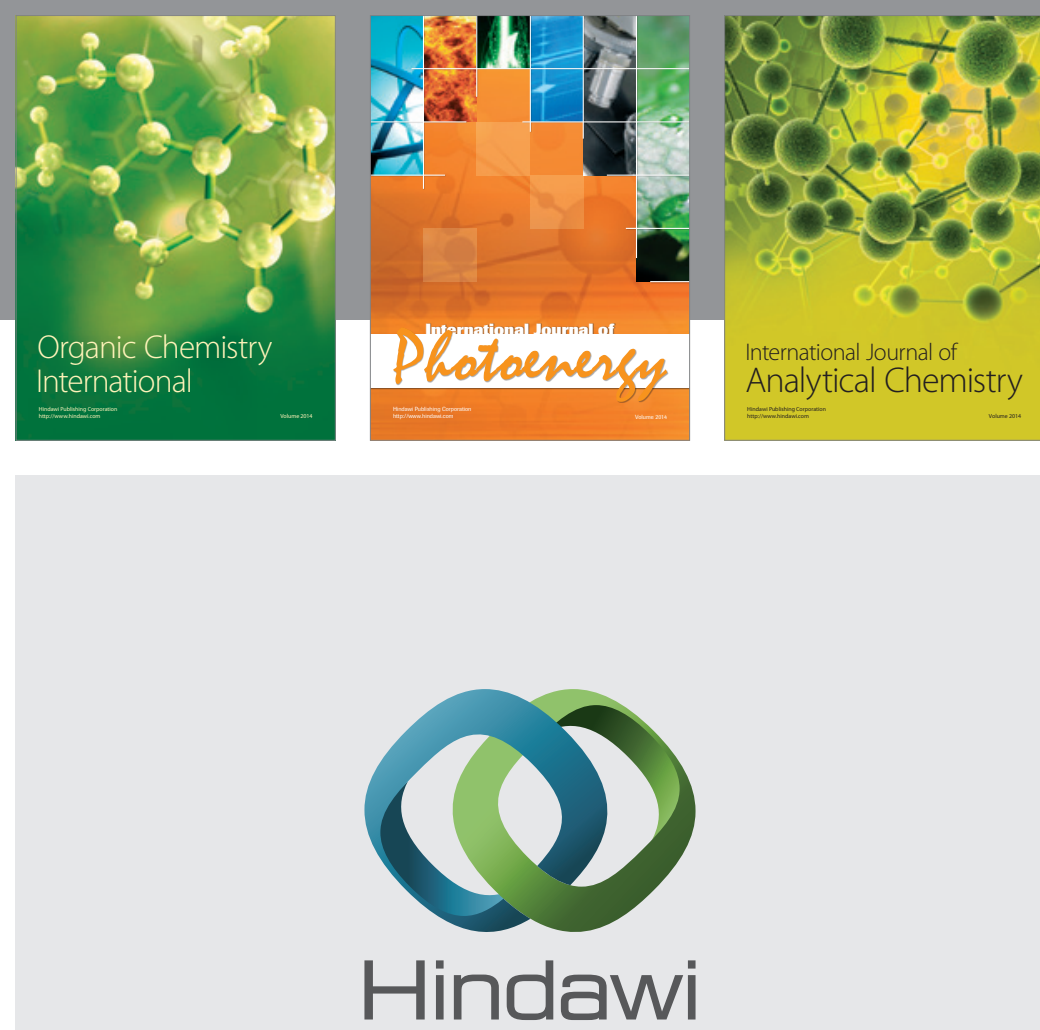

Submit your manuscripts at

http://www.hindawi.com
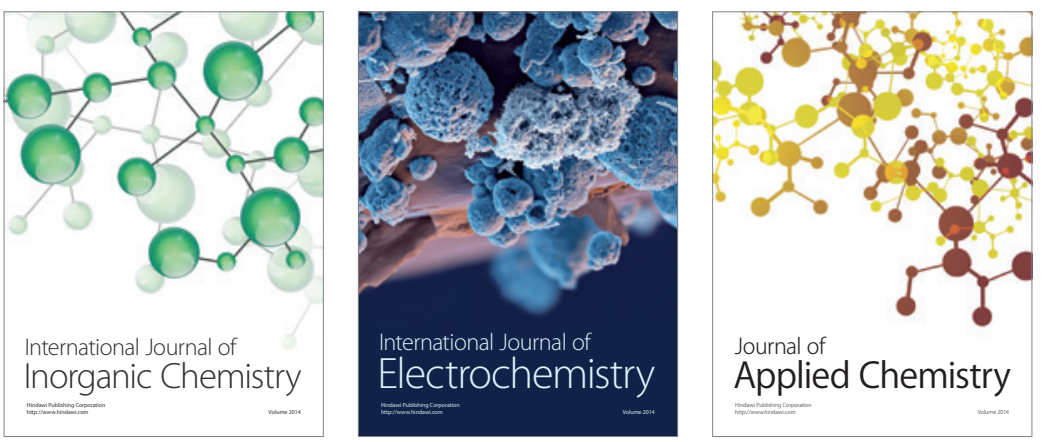

Journal of

Applied Chemistry
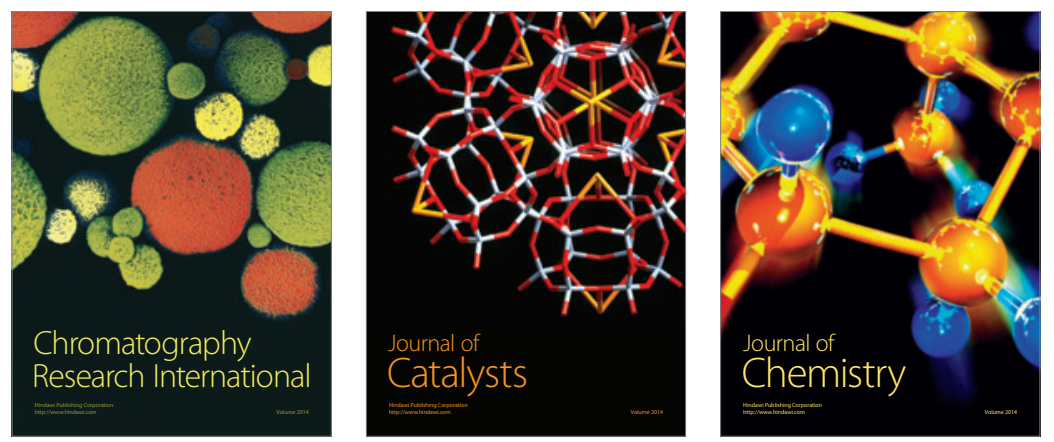
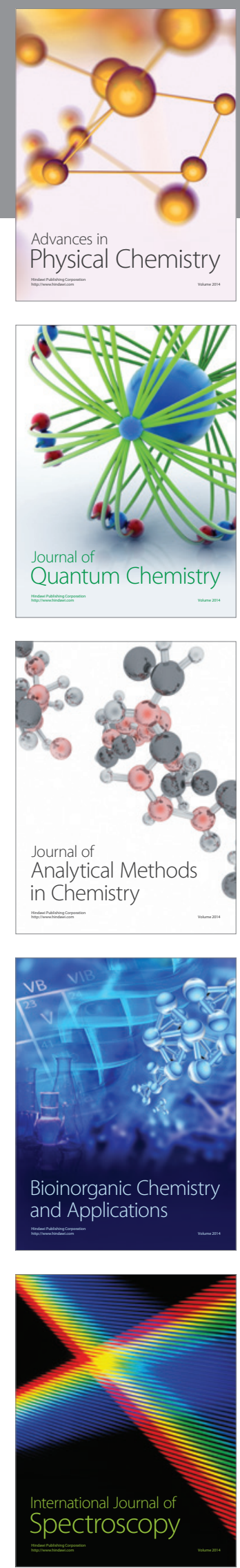